\title{
Original Research Article \\ Prognostic and theranostic role of epidermal growth factor receptor expression in colorectal cancers
}

\author{
Bindhuja J. ${ }^{1}$, Joseph L.D. ${ }^{2}$, Rajendiran S. ${ }^{3}$ \\ ${ }^{1}$ Dr. Bindhuja J, Assistant Professor, Sri Mookambika Institute of Medical Sciences, Kanyakumari, Tamil Nadu, \\ ${ }^{2}$ Dr. Leena Dennis Joseph, Professor, Sri Ramachandra Institute of Higher Education and Research, Chennai, Tamil \\ Nadu, ${ }^{3}$ Dr. Rajendiran S., Professor, Sri Ramachandra Institute of Higher Education and Research, Chennai, Tamil Nadu, \\ India.
}

Corresponding Author: Dr. Leena Dennis Joseph, Professor (Pathology), Sri Ramachandra Institute of Higher Education and Research, Chennai, India. E-mail: ID- leenadj@gmail.com

\begin{abstract}
Background: The outcome of Colorectal carcinoma (CRC) is highly influenced at diagnosis, presence of prognostic factors and the treatment modality. Evaluation of prognostic factors is predominantly done by pathological assessment of CRC resection specimens. This study was done to correlate the expression of EGFR in the colorectal carcinoma cases by immunohistochemistry which will facilitate the targeted therapy for patients suffering from this malignancy. Methods: This retrospective study was done on $\mathrm{H}$ and $\mathrm{E}$ sections retrieved from the surgical pathology registers on 100 cases of CRC. The methodology we used here were specimen reception, collection of clinical data, tissue processing $\&$ making paraffin blocks, hematoxylin \& Eosin staining, histological assessment, interpretation of data, selection of appropriate block for immuno histochemistry (IHC), IHC expression of EGFR in colonic carcinoma, correlation \& analysis of the above parameters for the final outcome. Results: The age of the patients ranged from 31 to 87 years and highest incidence was noted in the age group 41 to 51 years (32\%). The most common histological variants we analysed in our study were adenocarcinoma (78\%), mucinous (12\%), signet ring (22\%) and undifferentiated carcinoma (5\%). We noted that the staining of EGFR (IHC) had taken up only in the cytoplasm of all cases. Conclusion: The results of our study are in concordance with many studies in the literature. Even though EGFR is over expressed in various tumours it has only limited to no value in cancers of colon.
\end{abstract}

Key words: Adenocarcinoma, Colorectal cancers, Epidermal Growth Factor Receptor, Immunohistochemistry

\section{Introduction}

Colorectal carcinoma (CRC) remains to be one of the leading causes of cancer death in both men and women with an incidence varying from 3.7-0.7/100,000 among men and 3-0.4/100,000 among women [1]. The incidence rate is low in Indians as compared to others due to the high intake of fibre and natural antioxidants.

The outcome of CRC is highly influenced by the time at diagnosis, presence of prognostic factors and the treatment modality. Evaluation of prognostic factors is predominantly done by pathological assessment of CRC resection specimen, which includes the determination of staging, type and grade of the tumor, status of resected margins and presence of vascular invasion [2]. Although TNM classification is useful for staging patients in order to assign specific treatment, TNM

Manuscript received: $2^{\text {nd }}$ May 2019

Reviewed: $10^{\text {th }}$ May 2019

Author Corrected: $17^{\text {th }}$ May 2019

Accepted for Publication: $21^{\text {st }}$ May 2019 classification may not be a useful predictor to determine prognosis of the cancer. This warrants the need for biomarkers for evaluating the prognosis. Molecular markers have come to play a major role among which EGFR (epidermal growth factor) as a theranostic role on colonic carcinoma is worked up in our study.

Epidermal growth factor receptor (EGFR) and its downstream signalling pathways are involved in the development and progression of several human tumors including colorectal cancer [3]. EGFRs are considered as targets for anticancer therapy. In addition to playing a critical role in targeted therapy, alterations in this pathway can have prognostic implications. Mutations, gene amplifications and protein over-expression of various elements of this pathway impact prognosis and provide specific targets for therapeutic intervention. EGFR therefore has become an attractive target for therapy for CRC in two biologic agents- EGFR 
monoclonal antibodies and tyrosine kinase inhibitors $[4,5]$. The anti EGFR monoclonal antibodies and their predictive biomarkers have taken colon cancer treatment to the next level towards the goal of tailored cancer therapy [6].

With this background we focussed our study on evaluating the expression of EGFR in the colon carcinoma cases by Immunohistochemistry (IHC) and analysing its relationship with various histological and

\section{Original Research Article}

clinical parameters which facilitates the targeted therapy for patients suffering from this malignancy. The outcome of this study will help in substantiating the therapeutic benefit of anti-EGFR therapy for colorectal carcinoma patients in future.

Objectives- This study was carried out to evaluate the immunohistochemical expression of EGFR in colonic carcinoma tissues and its role as a prognostic and theranostic marker for CRC.

\section{Methodology}

Study setting- This study was carried out as a retrospective study by the Department of Pathology of Sri Ramachandra Institute of Higher Education and Research for a period of five years between 2009 and 2013.

Study participants- All the paraffin blocks of colectomy specimens received during the study period were taken up for the study. A total of 100 blocks of tumor areas along with adjacent normal areas of colostomy specimens were examined. Immunohistochemistry for EGFR was done on the sections along with controls.

Ethical approval- The study was approved by the Institutional Review Board of Sri Ramachandra Institute of Higher Education and Research (IRB No: CSP-MED/12/AUG/03/18.) and performed in accordance with the principles of the Declaration of Helsinki.

Data collection- Gross findings were recorded and clinical data was obtained from the medical records. Paraffin blocks, made after routine processing of the specimens were taken up for examination. Blocks with section containing normal epithelium and tumour on cases of H\&E proven colonic carcinoma were chosen for IHC study.

Hematoxylin and Eosin staining was done for microscopic analysis on resected lymph nodes and surgical margins. Other histopathological features observed by the investigator included intratumoral and peritumoral inflammatory response, adjacent dysplasia and adenomas.

Immunohistochemical staining for EGFR was performed by submitting the blocks with anti- EGFR primary antibody in dilution 1:30. Squamous cell carcinoma of cervix was taken as positive control. Positive controls were stained with every run. To avoid any potential interference sections were immunostained in batches and were all processed by a single experienced immuno-histotechnologist. EGFR expression by the immune-histochemical technique has been analysed in the colorectal carcinoma tissue, transitional tumor-mucosa, and in the normal mucosa, and hence correlated with clinicopathological aspects, clinical staging, metastases and in patients who had surgery for colorectal carcinoma.

Operational definition- A positive reaction was considered in H\&E examination when the colour Brown appeared on Membranous areas of the cell. The intensity of EGFR expression was recorded based on the criteria of Kountourakis $\mathrm{P}$ [7]. The grading of EGFR expression is given in table 1.

Table-1: Grading of intensity of EGFR expression among the samples:

\begin{tabular}{|c|c|c|}
\hline S. No & Grade & Interpretation \\
\hline 1 & 0 & Without staining \\
\hline
\end{tabular}

Positive staining of EGFR was defined as any membranous brown staining of malignant cells above background level. Cytoplasmic staining without associated membrane staining was considered as negative. The immunostaining results were recorded on a three-tier scale as negative (no staining), $1+$ (positivity in $<50 \%$ of cells) and $2+$ (positivity in $>50 \%$ of cells).

Data analysis-Data was entered and analyzed using Microsoft Excel Spreadsheet 2010. Data regarding the EGFR expression was presented as percentages. 


\section{Results}

\section{Original Research Article}

We studied 100 cases of histopathologically proven adenocarcinoma of colon diagnosed between January 2009 and June 2013. The age of the patients ranged from 31 to 87 years and highest incidence was noted in the age group 41 to 51 years (32\%), followed by the age group 51 to 60 years $(27 \%)$. This distribution pattern correlates with the age incidence. We also found that colon cancer occurred predominantly in men as compared to women with a male to female ratio of 2.1:1. In our study, $68 \%$ were males and $32 \%$ were females (Table 1 ).

Ascending colon is the most common site involved (36\%), followed by rectum (31\%) and sigmoid colon (12\%). The size of the tumour ranged between $1.5 \mathrm{~cm}$ to $16 \mathrm{~cm}$ and the highest ranged between 6 and $10 \mathrm{~cm}(49 \%)$, followed by size of more than $10 \mathrm{~cm}(37 \%)$. There is no definitive predilection of site for large tumors. (Table 2). The most common histological variants we analysed in our study were adenocarcinoma (78\%), mucinous (12\%), signet ring (22\%) and undifferentiated carcinoma (5\%). Grade I\& II tumours were categorized as low grade and grade III \& IV were categorized as high grade. In this study low gradetumors (grade 2) predominated (50\%) followed by grade 1 (35\%), irrespective of patients' gender.

Table-1: Background characteristics of the study participants:

\begin{tabular}{|c|c|c|c|}
\hline S. No & Characteristics & Frequency (N =100) & Percentage (\%) \\
\hline $\mathbf{1}$ & \multicolumn{3}{|c|}{ Age } \\
\hline & $<50$ years & 39 & 39.0 \\
\hline & $>50$ years & 61 & 61.0 \\
\hline $\mathbf{2}$ & Female & Sex & 32.0 \\
\hline & Male & 68 & 68.0 \\
\hline
\end{tabular}

Table-2: Tumor characteristics of the study participants.

\begin{tabular}{|c|c|c|c|}
\hline S. No & Characteristics & Frequency $(\mathrm{N}=100)$ & Percentage $(\%)$ \\
\hline 1 & \multicolumn{3}{|c|}{ Site } \\
\hline & Caecum & 10 & 10.0 \\
\hline & Ascending colon & 36 & 36.0 \\
\hline & Transverse & 10 & 10.0 \\
\hline & Descending colon & 1 & 1.0 \\
\hline & Sigmoid colon & 12 & 12.0 \\
\hline & Rectum & 31 & 31.0 \\
\hline \multirow[t]{4}{*}{2} & \multicolumn{3}{|c|}{ Size of Tumor } \\
\hline & $5 \mathrm{Cm}$ & 30 & 30.0 \\
\hline & $6-10 \mathrm{Cm}$ & 40 & 40.0 \\
\hline & $>10 \mathrm{~cm}$ & 30 & 30.0 \\
\hline \multirow[t]{5}{*}{3} & \multicolumn{3}{|c|}{ Histological type } \\
\hline & Mucinous & 12 & 12.0 \\
\hline & Adenocarcinoma & 77 & 77.0 \\
\hline & Signet ring & 6 & 6.0 \\
\hline & Undifferentiated carcinoma & 5 & 5.0 \\
\hline \multirow[t]{5}{*}{4} & \multicolumn{3}{|c|}{ Additional pathologic findings $(n=16)$} \\
\hline & High grade dysplasia & 5 & 31.2 \\
\hline & Colonic diverticuli & 1 & 6.2 \\
\hline & $\begin{array}{c}\text { Reactive lymphoid hyperplasia of } \\
\text { appendix }\end{array}$ & 6 & 37.5 \\
\hline & Granuloma & 4 & 25.1 \\
\hline
\end{tabular}


Table-3: Pathological findings.

\section{Original Research Article}

\begin{tabular}{|c|c|c|c|}
\hline S. No & Characteristics & Frequency $(N=100)$ & Percentage (\%) \\
\hline 1 & \multicolumn{3}{|c|}{ pT } \\
\hline & PT1 & 4 & 4.0 \\
\hline & PT2 & 33 & 33.0 \\
\hline & PT3 & 59 & 59.0 \\
\hline & PT4 & 3 & 3.0 \\
\hline & урТ2 & 1 & 1.0 \\
\hline 2 & \multicolumn{3}{|c|}{$\mathbf{N}$ break up } \\
\hline & N0 & 43 & 43.0 \\
\hline & N1 & 24 & 24.0 \\
\hline & $\mathrm{N} 2$ & 18 & 18.0 \\
\hline & $\mathrm{Nx}$ & 14 & 14.0 \\
\hline & YPNO & 1 & 1.0 \\
\hline 3 & \multicolumn{3}{|c|}{ M break up } \\
\hline & CM0 & 47 & 47.0 \\
\hline & CM1 & 2 & 2.0 \\
\hline & $\mathrm{MX}$ & 51 & 51.0 \\
\hline
\end{tabular}

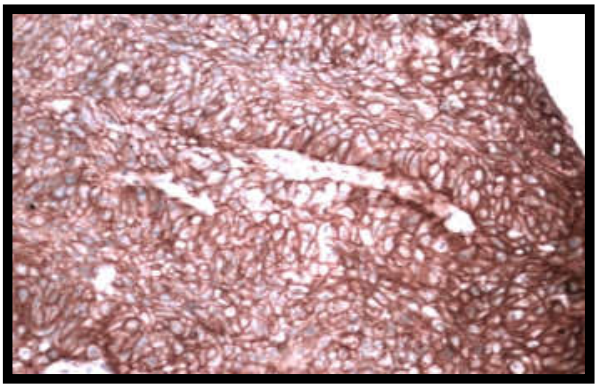

Fig-1: Control - Squamous cell carcinoma of cervix (Membrane positivity) IHC (x400)

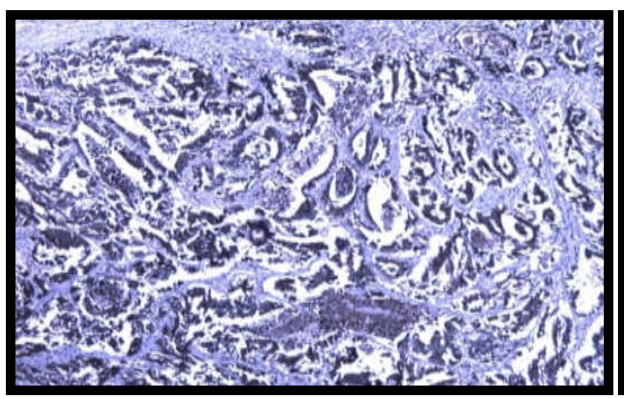

Fig-3: Poorly differentiated adenocarcinoma IHC EGFR- negative (x 100)

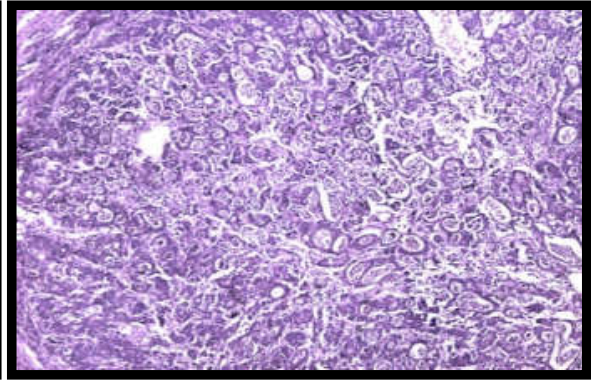

Fig-2: Poorly differentiated adenocarcinoma (H and E) x 100

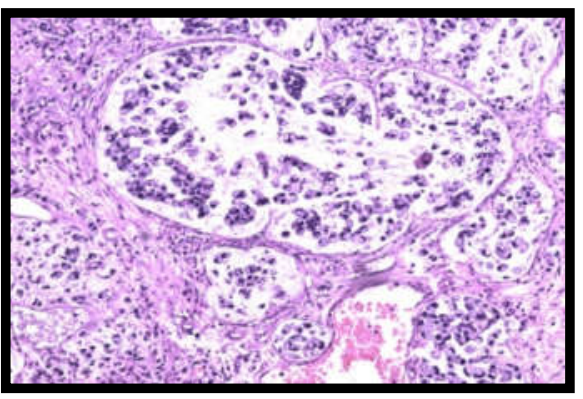

Fig-4: Signet ring adenocarcinoma $H$ and $E(x$ 100)

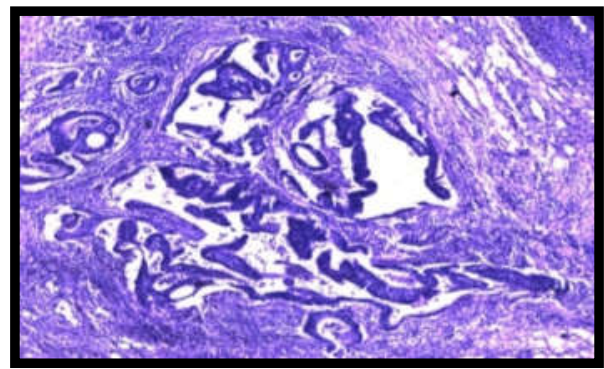

Fig-5: Lymphatic invasion $H$ and $E$ (x 100) 


\section{Original Research Article}

In our study most of the tumors (59\%) invaded through the muscularis propria into peri-colorectal tissues, classified as per TNM classification. About 33\% of the tumors invaded muscularis propria. With regards to the nodal staging, $43 \%$ of the patients did not show regional lumph node metastasis, while $24 \%$ showed metastasis in one or more regional lymph node, while $18 \%$ showed metastasis in more than 4 lymph nodes. Clinically no distant metastasis (cM0) was noted in of cases. (Table 3)

Finally we analysed the expression of EGFR in normal colonic epithelium, dysplastic epithelium and carcinoma and hence correlated with its expression. We noted that the staining of EGFR (IHC) had taken up only in the cytoplasm of all cases (normal colonic mucosa, moderately differentiated adenocarcinoma, poorly differentiated, mucinous, signet ring) Figures 1-4. Control was taken up from the squamous cell carcinoma of cervix (membrane positivity) The additional pathological findings we noted among all the 100 cases were reactive lymphoid hyperplasia of appendix (6\%), high grade dysplasia (5\%), tubulovillous adenoma(Figure 5)

\section{Discussion}

Colorectal carcinoma is the most common malignancy of the gastrointestinal tract. More than $78 \%$ of the cancers of the colorectal region are adenocarcinomas as per our study. According to Carolyn et al, colon cancer patients were reported to have a higher rate of adenocarcinoma (92\%) and this finding correlates with our data [8]. Males (68\%) were affected more than females $(32 \%)$. In a case study by Lyon et al, the relative rate of sex incidence were at a higher level in men (3.6) when compared to women (2.5)[9]. The incidence rate peaks at 35 to 82 years of age in our study. The mean age at the time of diagnosis was 58 years (in the range of 25-86 yrs) in a study by Oliver et al, which is similar to our study [10].

In our study ascending colon was the most common site for colorectal adenocarcinoma followed by rectum irrespective of age groups. In our study right sided lesions were higher and were more likely to present at an older age than left sided lesions; and left sided lesions on the other hand have a greater chance of presenting with bleeding per rectum and changes in bowel habits. Similar observations were seen in studies done by $\mathrm{Wu}$ Zhang et al [11]. Studies have shown that tumour size is of limited prognostic significance. In our study the highest range of size of tumors varies between 6-10 cm (49\%).

As a general rule the degree of gland formation $(50 \%$ cut off) is widely regarded as the most important feature in grading. The colorectal carcinoma has been graded as well differentiated (grade I), moderately differentiated (grade II) and poorly differentiated (grade III). However, Grade IV has been considered redundant as a category that shows no histological evidence of differentiation and is classified as a separate histological type. As per our study low grade tumors have a higher prevalence rate $(50 \%)$. Despite the lack of standardization, inter observer variation in the assessment of histological grade has been shown repeatedly by multivariate analysis.
The pathological features of resected specimen constitute the most powerful predictors of post operative outcome which includes prognostic factors such as TNM staging, histologic grade, status of resected margins, vascular invasion and perineural invasion.

The expression of EGFR marker in this study was significantly expressed as only cytoplasmic positivity in the neoplastic tissue, normal colonic epithelium, stromal cells. Also, this study observed that the measures of immunoexpression (intensity of expression) presented significant differences in the tumor cells, transitional tumor-mucosa and adjacent normal mucosa. Considering that the immunoexpression has a direct relation with the amount of EGFR in the sample tissue, it suggests that the content of EGFR is more intense in the neoplastic tissue when compared to the adjacent non-neoplastic mucosa.

Our study also showed that EGFR expression was similar in well, moderately and poorly differentiated colorectal carcinomas. Additional studies are important to analyze the relation between immunohistochemical expression of EGFR and the prognosis of colorectal carcinoma, and also if the immunohistochemical expression of EGFR can be used as a predictive marker so that the patient has positive results with chemotherapy.

Mutations involving EGFR leads to constant activation, which could result in uncontrolled cell division that predisposes to cancer. Consequently, mutations of EGFR have been identified in several types of cancer, and it is the target of an expanding class of anticancer therapies (cetuximab). Preliminary data suggest that recurrences following postoperative chemotherapy are likely to have lower levels of EGFR expression compared to cases who receive no chemotherapy. Although the difference of immunostaining profiles was not statistically significant, this observation might 


\section{Original Research Article}

impact the management of these patients by targeted biologic therapies and its practical implications need further validation in larger series. There is immunohistochemical evidence to suggest that expression of epidermal growth factor receptor (EGFR) in primary colorectal adenocarcinoma predicts its expression in recurrent disease [12--16].

Important observation of Atkins et alis that immunoreactivity of anti EGFR is inversely correlated with storage time, with a substantial drop in the number of 3 + EGFR readings to a substantial increase in the number of EGFR negative/zero, readings with an increase in storage time from 3 to 24 months, and even this degree of variation varied considerably depending on which fixative had been used [16].

Cytoplasmic staining alone is commonly seen; however, the test should be repeated because at times, diffuse cytoplasmic staining makes it difficult to distinguish the diagnostic membrane staining and interpret the results. Positive tissue controls are indicative of correctly prepared tissues and proper staining techniques. One positive tissue control for each set should be included in each staining run. The tissues used for the positive tissue controls should give weak positive staining so they can detect subtle changes in the primary antibody sensitivity. The Control Slides supplied with this system or specimens processed differently from the patient sample (s) validate reagent performance only and do not verify tissue preparation. If the positive tissue controls fail to demonstrate appropriate positive staining, results with the test specimens should be considered invalid. In our study as a result of cytoplasmic positivity alone in view of confirming our results; we repeated the stain of EGFR in few of our cases by sending our slides to Hyderabad and SRM university ; where also the cases were interpreted as negative (cytoplasmic positivity alone).

\section{Conclusion}

The effectiveness of EGFR expression against colorectal carcinoma has to be further evaluated using molecular studies. Efforts to evaluate the histological features have limited success, although microsatellite instability shows a modest correlation with the same. EGFR in our case as a prognostic marker, and its over expression was proved to correlate with poor prognosis. We conclude that in our study EGFR over expression has been cytoplasmic positivity only and of no significance; so it was concluded to be negative. EGFR has no theranostic effect and hence its effectiveness against colorectal carcinoma has to be evaluated using molecular studies.
What this study adds to existing knowledge? Our study has elucidated the role of EGFR in predicting the prognosis. The EGFR over expression has been witnessed in the cytoplasm although it was not statitistically significant.

\section{Author contribution}

Conceptualization: BJ, Data curation: BJ, Formal analysis: $\mathrm{BJ}, \mathrm{LDJ}$

Investigation: BJ, Methodology: LDJ. RS, Project administration: LDJ, RS, Resources: LDJ, Software: LDJ, Supervision: LDJ, RS, Validation: RS, Visualization: BJ, LDJ, Writing-original draft: BJ, Writing- review \& editing: LDJ, RS

Findings: Nil; Conflict of Interest: None initiated Permission from IRB: Yes

\section{References}

1. Haggar FA, Boushey RP. Colorectal cancer epidemiology: incidence, mortality, survival, and risk factors. Clin Colon Rectal Surg. 2009 Nov;22(4):191-7. doi: $10.1055 / \mathrm{s}-0029-1242458$.

2. Fleming M, Ravula S, Tatishchev SF, et al. Colorectal carcinoma: Pathologic aspects. J Gastrointest Oncol. 2012 Sep;3(3):153-73. doi: 10.3978/j.issn.20786891.2012.030.

3. Sasaki T, Hiroki K, Yamashita Y. The role of epidermal growth factor receptor in cancer metastasis and microenvironment. Biomed Res Int. 2013;2013: 546318. doi: 10.1155/2013/546318. Epub 2013 Aug 7.

4. Hoda D, Simon GR, Garrett CR. Targeting colorectal cancer with anti-epidermal growth factor receptor antibodies: focus on panitumumab. Ther Clin Risk Manag. 2008 Dec;4(6):1221-7.

5. Yarom N, Jonker DJ. The role of the epidermal growth factor receptor in the mechanism and treatment of colorectalcancer.DiscovMed.2011Feb;11(57):95-105

6. Chen D, Li L, Zhang X, et al. FOLFOX plus antiepidermal growth factor receptor (EGFR) monoclonal antibody $(\mathrm{mAb})$ is an effective first-line treatment for patients with RAS-wild left-sided metastatic colorectal cancer: A meta-analysis. Medicine (Baltimore). 2018 Mar;97(10):e0097.doi:10.1097/MD.0000000000010097

7. Kountourakis P, Pavlakis K, Psyrri A, Rontogianni D, Xiros N, Patsouris E, et al. Clinicopathologic significance of EGFR and Her-2/neu in colorectal adenocarcinomas. Cancer J 2006;12(3):229-36 
8. Nelson H, Petrelli N, Carlin A, et al. Guidelines 2000 for colon and rectal cancer surgery. J Natl Cancer Inst. 2001 Apr 18;93(8):583-96.

9. Jemal A, Siegel R, Ward E, et al. Cancer statistics, 2006. CA Cancer J Clin. 2006 Mar-Apr;56(2):106-30.

10. Chok KS, Law WL. Prognostic factors affecting survival and recurrence of patients with pT1 and pT2 colorectal cancer. World J Surg 2007;31(7):1485-90.

11. Zhang H, Berezov A, Wang Q, et al. ErbB receptors: from oncogenes to targeted cancer therapies. J Clin Invest. 2007 Aug;117(8):2051-8.

12. Galizia G, Lieto E, Ferraraccio F, et al. Prognostic significance of epidermal growth factor receptor expression in colon cancer patients undergoing curative surgery. Ann Surg Oncol. 2006 Jun;13(6):823-35. Epub 2006 Apr 18.

\section{Original Research Article}

13. Rego RL, Foster NR, Smyrk TC, et al. Prognostic effect of activated EGFR expression in human colon carcinomas: comparison with EGFR status. $\mathrm{Br} \mathrm{J}$ Cancer. 2010 Jan 5;102(1):165-72. doi: 10.1038/sj.bjc. 6605473. Epub 2009 Dec 8.

14. Juan Rosai. Gastrointestinal tract - Large bowel, Rosai and Ackerman's Surgical Pathology,10th Edition, Missouri, Elsevier, 2012; 731-802.

15. Spano JP, Lagorce C, Atlan D, et al. Impact of EGFR expression on colorectal cancer patient prognosis and survival. Ann Oncol. 2005 Jan;16(1):102-8.

16. Atkins D, Reiffen KA, Tegtmeier CL, et al. Immunohistochemical detection of EGFR in paraffinembedded tumor tissues: variation in staining intensity due to choice of fixative and storage time of tissue sections. J Histochem Cytochem. 2004 Jul; 52 (7): 893-901.

\section{How to cite this article?}

Bindhuja J, Joseph L.D, Rajendiran S. Prognostic and theranostic role of epidermal growth factor receptor expression in colorectal cancers. Trop J Path Micro 2019;5(5):331-337. doi: 10.17511/jopm.2019.i05.12. 